\title{
Real-Time System for Monitoring Driver Vigilance
}

\author{
Luis M. Bergasa, Associate Member, IEEE, Jesús Nuevo, \\ Miguel A. Sotelo, Member, IEEE, Rafael Barea, and María Elena Lopez
}

\begin{abstract}
This paper presents a nonintrusive prototype computer vision system for monitoring a driver's vigilance in real time. It is based on a hardware system for the real-time acquisition of a driver's images using an active IR illuminator and the software implementation for monitoring some visual behaviors that characterize a driver's level of vigilance. Six parameters are calculated: Percent eye closure (PERCLOS), eye closure duration, blink frequency, nodding frequency, face position, and fixed gaze. These parameters are combined using a fuzzy classifier to infer the level of inattentiveness of the driver. The use of multiple visual parameters and the fusion of these parameters yield a more robust and accurate inattention characterization than by using a single parameter. The system has been tested with different sequences recorded in night and day driving conditions in a motorway and with different users. Some experimental results and conclusions about the performance of the system are presented.
\end{abstract}

Index Terms-Driver vigilance, eyelid movement, face position, fuzzy classifier, percent eye closure (PERCLOS), visual fatigue behaviors.

\section{INTRODUCTION}

$\mathbf{T}$ HE INCREASING number of traffic accidents due to a driver's diminished vigilance level has become a serious problem for society. In Europe, statistics show that between $10 \%$ to $20 \%$ of all traffic accidents are due to drivers with a diminished vigilance level caused by fatigue. In the trucking industry, about $60 \%$ of fatal truck accidents are related to driver fatigue. It is the main cause of heavy truck crashes [1].

According to the U.S. National Highway Traffic Safety Administration (NHTSA), falling asleep while driving is responsible for at least 100000 automobile crashes annually. An annual average of roughly 40000 nonfatal injuries and 1550 fatalities result from these crashes [2]. These figures only cover crashes happening between midnight and 6 a.m., involving a single vehicle and a sober driver traveling alone, including the car departing from the roadway without any attempt to avoid the crash. These figures underestimate the true level of the involvement of drowsiness because they do not include crashes during daytime hours involving multiple vehicles, alcohol, passengers, or evasive maneuvers. These statistics do not deal with crashes caused by driver distraction, which is believed to be a larger problem. As car manufacturers incorporate intelligent vehicle

Manuscript received December 7, 2004; revised July 27, 2005 and October 25, 2005. This work was supported by Grant FOM2002-002 from the Spanish Ministry of Public Works and by Grant DPI2002-02193 Robotic System for Elderly Assistance (SIRAPEM Project) from the Spanish Ministry of Science and Technology (MCyT). The Associate Editor for this paper was T. A. Dingus.

The authors are with the Department of Electronics, University of Alcala, Madrid 28805, Spain (e-mail: bergasa@ depeca.uah.es; jnuevo@depeca.uah.es; sotelo@depeca.uah.es; barea@depeca.uah.es; elena@depeca.uah.es).

Digital Object Identifier 10.1109/TITS.2006.869598 systems in order to satisfy the consumer's ever increasing demand for a wired, connected world, the level of cognitive stress on drivers is also increased. That is, the more assistant systems for comfort, navigation, or communication, the more sources of distraction from the most basic task at hand, i.e., driving the vehicle.

With this background, developing systems for monitoring the driver's level of vigilance and alerting the driver when he is not paying adequate attention to the road is essential in order to prevent accidents. This paper presents an original system for monitoring the driver's inattention, focusing on the drowsiness or fatigue category according to the classification shown in [3].

The rest of the paper is arranged as follows. In Section II, we present a review of previous studies in this line. Section III describes the general system architecture, explaining its main parts. Experimental results are shown in Section IV. Finally, we present our conclusions and future studies in Section V.

\section{Previous Studies}

In the past few years, many researchers have been working on the development of safety systems using different techniques. The most accurate techniques are based on physiological measures like brain waves, heart rate, pulse rate, respiration, etc. However, these techniques are intrusive since they require electrodes to be attached to the drivers, causing annoyance to them. A representative project in this line is the MIT Smart Car [4], where several sensors (electrocardiogram, electromyogram, respiration, and skin conductance) are embedded in a car and visual information for sensor confirmation are used. In the advanced safety vehicle (ASV) project conducted by Toyota [5], the driver must wear a wristband in order to measure his heart rate. Others techniques monitor eyes and gaze movements using a helmet or special contact lenses [6]. These techniques, though less intrusive, are still not acceptable in practice.

A driver's state of vigilance can also be characterized by indirect vehicle behaviors like the lateral position, steering wheel movements, and time-to-line crossing. Although these techniques are not intrusive, they are subject to several limitations such as vehicle type, driver experience, geometric characteristics, condition of the road, etc. On the other hand, these procedures require a considerable amount of time to analyze user behaviors and therefore, they do not work with the socalled microsleeps-when a drowsy driver falls asleep for a few seconds on a very straight road section without changing the lateral position of the vehicle [7]. In this line, we can find different experimental prototypes, but at this moment none of them has been commercialized. Among these prototypes, there is an important Spanish system called tech co driver 
(TCD) [8] which is based on steering wheel and lateral position sensors. Toyota [5] uses steering wheel sensors (steering wheel variability) and a pulse sensor to record the heart rate, as explained above. Mitsubishi has reported the use of steering wheel sensors and measures of vehicle behavior (such as the lateral position of the car) to detect the driver's drowsiness in their ASV system [5]. Daimler Chrysler has developed a system based on vehicle speed, steering angle, and vehicle position relative to road delimitation (recorded by a camera) to detect if the vehicle is about to leave the road [9].

People experiencing fatigue show some easily observable visual behaviors from the changes in their facial features like the eyes, head, and face. Typical visual characteristics observable from the images of a person with a reduced alertness level include a longer blink duration, slow eyelid movement, smaller degree of eye opening (or even closed), frequent nodding, yawning, gaze (narrowness in the line of sight), sluggish facial expression, and drooping posture. Computer vision can be a natural and nonintrusive technique for extracting visual characteristics that typically characterize a driver's vigilance from the images taken by a camera placed in front of the user. Many studies on developing image-based driver alertness using computer vision techniques have been reported in the literature. Some of them focus primarily on head and eye tracking techniques using two cameras. The method presented in [10] estimates the head pose and gaze direction. It relies on a two-dimensional (2-D) template searching and then a threedimensional (3-D) stereo matching of the facial features. A 3-D model is then fit and minimized using virtual springs, instead of the least-squares fit approach for determining the head pose. In [11], a method is presented based on a stereo template matching system to determine some specific facial features. A leastsquares optimization is done to determine the exact pose of the head. Two eye trackers calculate the eye-gaze vector for each eye; these vectors are combined with the head pose to determine the gaze direction. In [12], a system called FaceLAB developed by a company called Seeing Machines is presented. This is an evolution of the two previous studies. The 3-D pose of the head and the eye-gaze direction are calculated in an exact way. FaceLAB also monitors the eyelids to determine eye opening and blink rates. With this information, the system estimates the driver's fatigue level. The system operates at both day and night, but at night the performance of the system decreases. All the abovementioned systems rely on a manual initialization of the feature points. The systems appear to be robust, but manual initialization is a limitation, although it makes the whole problem of tracking and pose estimation trivial.

In [13], we can find a 2-D pupil monocular tracking system based on the differences in the color and reflectivity between the pupil and iris. The system monitors driving vigilance by studying the eyelid movement. Another successful head/eye monitoring and tracking system that can detect the drowsiness of drivers using one camera and based on color predicates is presented in [14]. However, this system is based on passive vision techniques and its functionality can be problematical in poor or very bright lighting conditions. Moreover, it does not work at night, when the monitoring is more important.
In order to work at night, some studies use active illumination based on infrared LEDs. In [15], a system using 3-D vision techniques to estimate and track the 3-D line of sight of a person using multiple cameras is proposed. The method relies on a simplified eye model and uses the Purkinje images of an infrared light source to determine the eye location. With this information, the gaze direction is estimated. However, nothing about monitoring driver vigilance is presented. In [16], a system with active infrared LED illumination and a camera is implemented. Because of the LED illumination, the method can easily find the eyes and based on them, the system locates the rest of the facial features. The authors in [16] propose to analytically estimate the local gaze direction based on the pupil location. They calculate the eyelid movement and face orientation to estimate driver fatigue. Almost all the active systems reported in the literature have been tested in simulated environments, but not in real moving vehicles. A moving vehicle presents new challenges like variable lighting, changing background, and vibrations that must be taken into account in real systems. In [17], an industrial prototype called Copilot is presented. This system uses infrared LED illumination to find the eyes and has been tested with truck drivers in real environments. It uses a simple subtraction process for finding the eyes and it only calculates a validated parameter called the percent eye closure (PERCLOS) in order to measure a driver's drowsiness. This system currently works under low light conditions.

Systems relying on a single visual cue may encounter difficulties when the prerequisite visual features cannot be acquired accurately or reliably, which happens in real conditions. Therefore, a single visual cue may not always be indicative of the overall mental condition [16]. The use of multiple visual cues reduces the uncertainty and the ambiguity present in the information from a single source. Most recent studies in this line use this hypothesis. Recently, the ambitious European project, system for effective Assessment of driver vigilance and Warning According to traffic risK Estimation (AWAKE) [1] was developed. The consortium includes two major car manufacturers (Fiat, DaimlerChrysler), four automotive system developers [Siemens, Association de Coordination Technique pour l'Industrie Agro-Alimentaire (ACTIA), Navigation Technologies (NAVTECH), and Autoliv] and many research institutes and universities. A multisensor approach is proposed in this project, adapted to the driver, the vehicle, and the environment in an integrated way. This system merges data from on-board driver monitoring sensors (such as an eyelid camera and a steering grip sensor) as well as driver behavior data (i.e., from the lane tracking sensor, gas/brake, and steering wheel positioning) via an artificial intelligent algorithm. The system must be personalized for each driver during the learning phase. The system has undergone exhaustive pilot testing to determine its functional performance and the user acceptance of the application [18]. The final results of this project conclude that this system cannot be used outside the well-structured highway scenario nor can be applicable for all drivers and that further research is necessary.

This paper describes a real-time prototype system based on computer vision for monitoring driver vigilance using active infrared illumination and a single camera placed on the car 


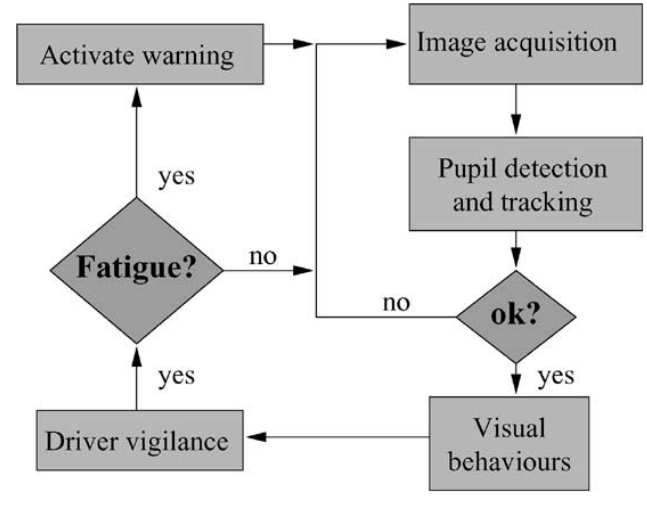

Fig. 1. General architecture.

dashboard. We have employed this technique because our goal is to monitor the driver in real conditions (vehicle moving) and in a very robust and accurate way, mainly at night (when the probability to crash due to drowsiness is at the highest). The proposed system does not require manual initialization and monitors several visual behaviors that typically characterize a person's level of alertness while driving. In a different manner than other previous studies, we have fused different visual cues from one camera using a fuzzy classifier instead of different cues from different sensors. We have analyzed different visual behaviors that characterize a drowsy driver and we have studied the best fusion for optimal detection. Moreover, we have tested our system in the duration of several hours in a car moving in a motorway and with different users. The preliminary result of this system was presented in [19].

\section{System ARChitecture}

The general architecture of our system is shown in Fig. 1. It consists of four major modules: 1) image acquisition; 2) pupil detection and tracking; 3) visual behaviors; and 4) driver vigilance. The image acquisition is based on a lowcost charge-coupled device (CCD) microcamera sensitive to near IR. The pupil detection and tracking stage is responsible for segmentation and image processing. The pupil detection stage is simplified by the "bright pupil" effect, similar to the red-eye effect in photography. We then use two Kalman filters in order to track the pupils robustly in real time. In the visual behavior stage, we calculate some parameters from the images in order to detect some visual behaviors easily observable in people experiencing fatigue: slow eyelid movement, smaller degree of eye opening, frequent nodding, blink frequency, and face pose. Finally, in the driver vigilance evaluation stage, we fuse all individual parameters obtained in the previous stage using a fuzzy system, yielding the driver inattentiveness level. An alarm is activated if this level exceeds a certain threshold.

\section{A. Image Acquisition System}

The purpose of this stage is to acquire video images of the driver's face. In this application, the acquired images should be relatively invariant to light conditions and should facilitate

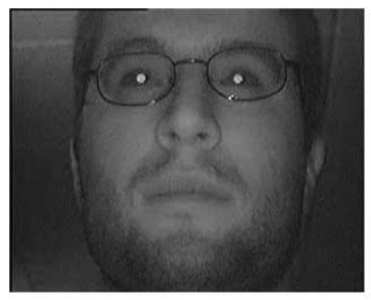

(a)

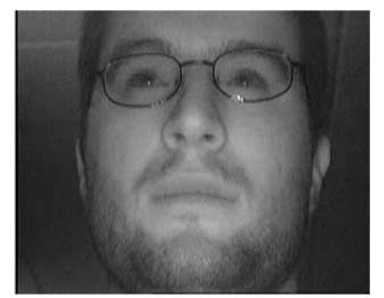

(b)

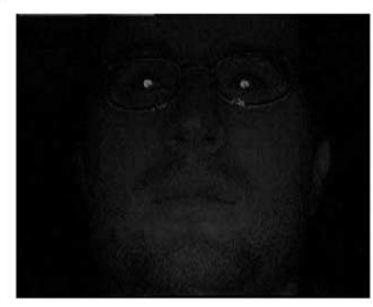

(c)

Fig. 2. Fields captured and subtraction. (a) Image obtained with inner IR ring. (b) Image obtained with outer IR ring. (c) Difference Image.

the eye detection and tracking (good performance is necessary). The use of a near-IR illuminator to brighten the driver's face serves these goals [20]. First, it minimizes the impact of changes in the ambient light. Second, the near-IR illumination is not detected by the driver and does not interfere with the user's driving. Third, it produces the bright pupil effect, which constitutes the foundation of our detection and tracking system. A bright pupil is obtained if the eyes are illuminated with an IR illuminator beaming light along the camera's optical axis. At the IR wavelength, the retina reflects almost all of the IR light received along the path back to the camera, and a bright pupil effect is produced in the image. When illuminated off the camera optical axis, the pupils appear to be dark since the reflected light of the retina does not enter the camera lens. An example of the bright/dark pupil effect can be seen in Fig. 2. This pupil effect is clear with and without glasses, with contact lenses, and it even works to some extent with sunglasses.

Fig. 3 shows the image acquisition system configuration. It is composed by a miniature CCD camera sensitive to near IR and is located on the dashboard of the vehicle. This camera focuses on the driver's head for detecting the multiple visual behaviors. The IR illuminator is composed of two sets of IR LEDs distributed symmetrically along two concentric and circular rings. An embedded PC with a low-cost frame grabber is used for the video signal acquisition and signal processing. The image acquisition from the camera and the LED excitation is synchronized. The LED rings illuminate the driver's face alternatively, one for each of the image fields, providing different lighting conditions for almost the same image once the fields are deinterlaced on the next stages.

The ring sizes have been empirically calculated in order to obtain a dark pupil image if the outer ring is turned on and a bright pupil image if the inner ring is turned on. The LEDs in the inner ring are as close as possible to the camera in order to maximize the "bright pupil" effect. The value of the outer ring radius is a compromise between the resulting illumination, 


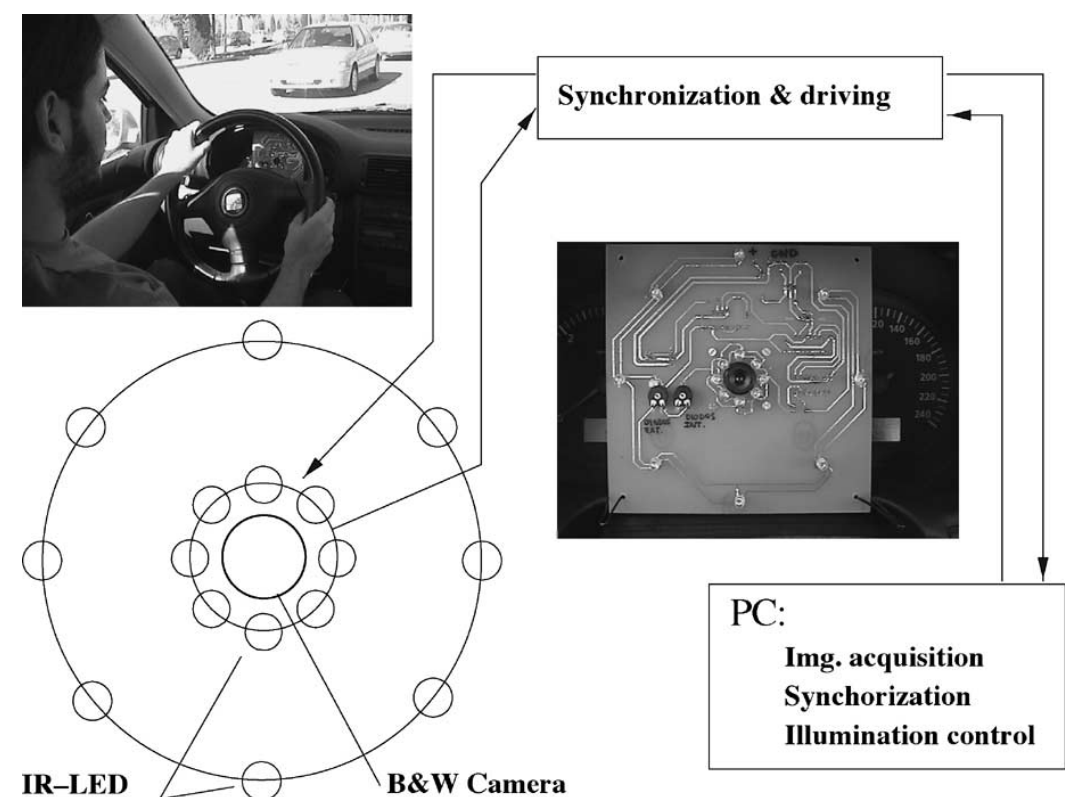

Fig. 3. Block diagram of the prototype.

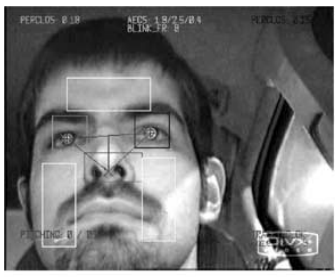

(a)

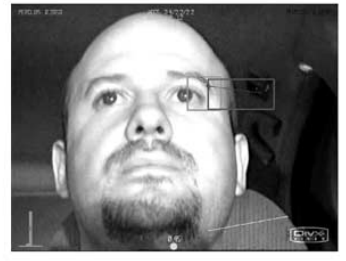

(b)

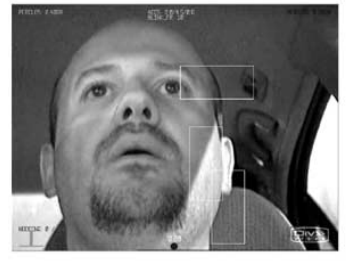

(c)

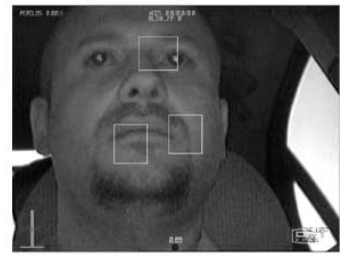

(d)

Fig. 4. Effects of external lights on the acquisition system. (a) Out-of-the-road lights effect. (b) Vehicle lights effect. (c) Sunlight effect. (d) Sunlight effect with filter.

which improves as it is increased, and the available space in the car's dashboard. The symmetric position of the LEDs in the rings around the camera optical axis cancels the shadows generated by the LEDs. The inner ring configuration obtains the bright pupil effect because the center of the ring coincides with the camera's optical axis, actuating as if there were only an LED located on the optical axis of the lens. The outer ring provides an ambient illumination that is used for contrast enhancing. Despite of the LEDs producing the dark pupil effect, a glint can be observed on each pupil.

The abovementioned acquisition system works very well under controlled light conditions, but real scenarios present new challenges that must be taken into account. Lighting conditions are one of the most important problems to be solved in real tests. As our system is based on the reflection of the light emitted by the IR LEDs, external light sources are the main source of noise. Three main sources can be considered, as depicted in Fig. 4-artificial light from elements outside the road (such as light bulbs), vehicle lights, and sunlight. The effect of lights from elements outside the road mainly appears in the lower part of the image [Fig. 4(a)] because they are situated above the height of the car and the beams enter the car with a considerable angle. However, this noise can be easily filtered. On the other hand, when driving on a double direction road, vehicle lights directly illuminate the driver, increasing the pixel levels quickly and causing the pupil effect to disappear [Fig. 4(b)]. Once the car has passed, the light level declines quickly. Only after a few frames, the automatic gain controller (AGC) integrated in the camera compensates the changes, so very light and dark images are obtained, affecting the performance of the inner illumination system.

Regarding the sunlight, it only a factor during daytime but its effect changes as function of the weather (sunny, cloudy, rainy, etc.) and the time of the day. With the exception of sunset, dawn, and cloudy days, sunlight hides the inner IR illumination and then the pupil effect disappears [Fig. 4(c)]. In order to minimize the interference from light sources beyond the IR light emitted by our LEDs, a narrow bandpass filter centered at the LED wavelength has been attached between the CCD and the lens. This filter solved the problem of artificial lights and vehicle light almost completely, but it adds a new drawback for it reduces the intensity of the image and the noise is considerably amplified by the AGC. The filter does not eliminate the sunlight interference, except for cases when the light intensity is very low. This is caused by the fact that the power emitted by the sun in the band of the filter is able to hide the inner illumination. An image of this case taken during sunset is depicted in Fig. 4(d). A possible solution to 
this problem could be the integration of IR filters in the car's glasses. This option does not have been tested yet.

\section{B. Pupil Detection and Tracking}

This stage starts with the pupil detection. As mentioned above, each frame is deinterlaced in even and odd fields, containing the bright and dark pupil images separately. The even image field is then digitally subtracted from the odd image field to produce the difference image. In this image, the pupils appear as the brightest parts in the image, as can be seen in Fig. 2. This method minimizes the ambient light influence by subtracting it in the generation of the difference image. This procedure yields high contrast images where the pupils are easily found. It can be observed that the glint produced by the outer ring usually falls close to the pupil, with the same gray level as the bright pupil. The shape of the pupil blob in the difference image is not a perfect ellipse because the glint cuts the blob, affecting the modeling of the pupil blobs and, consequently, the calculation depending on it, as will be explained later. This is the reason why the system only uses subtracted images during initialization and when light conditions are poor (this initialization time varies depending on the driver and light conditions, but it was below $5 \mathrm{~s}$ for all tests). In other cases, only the field obtained with the inner ring is processed, increasing accuracy and reducing the computation time.

The pupils are detected on the resulting image by searching the entire image to locate two bright blobs that satisfy certain constraints. The image is binarized using an adaptive threshold for detecting the brighter blobs in the image.

A standard eight-connected-components analysis is then applied to the binaryzed difference image to identify the binary blobs that satisfy certain size and shape constraints. The blobs that are outside of some size constraints are removed; for others, an ellipse model is fit to each one. The best candidates are selected depending on their size, intensity, position and distance and all the possible pairs between them are evaluated. The pair with the highest qualification is chosen as the detected pupils and its centroids are returned as the pupil positions.

One of the main characteristics of this stage is that it is applicable to any user without any supervised initialization. Nevertheless, the reflection of the IR in the pupils under the same conditions varies from one driver to another. Even on the same driver, the intensity depends on the gaze point, head position, and the opening of the eye. Apart from those factors, lightning conditions change with time, which modifies the intensity of the pupils. On other hand, the size of the pupils also depends on the user and the distance to the camera. To deal with those differences, in order to be generic, our system uses an adaptive threshold in the binarization stage. The parameters of the detected pupils are used to update the statistics that set the thresholds and margins in the detection process. Those statistics include size, gray level, position and the apparent distance, and the angle between pupils, calculated over a time window of $2 \mathrm{~s}$. The values of the thresholds are modified if the pupils are not found, widening the margins to make more candidates available to the system.
Another question related to illumination that is not usually addressed in the literature is the sensitivity of the eye to the IR emission. As the exposure time to the IR source increases, its power has to be reduced in order to avoid damaging the internal tissues of the eye. This imposes a limit on the emission of the IR LEDs. To calculate the power of our system, we have followed the recommendations in [21], based on the International Electrotechnical Commission (IEC) 825-1 and the European Committee for Electrotechnical Standardization (CENELEC) 60825-1 infrared norms. With these limitations, no negative effects have been reported in the drivers that participated in the tests.

To continuously monitor the driver, it is important to track his pupils from frame to frame after locating the eyes in the initial frames. This can be done efficiently by using two Kalman filters, one for each pupil, in order to predict the pupil positions in the image. We have used a pupil tracker based on [16] and we have tested it with images obtained from a car moving in a motorway. The Kalman filters presented in [16] work reasonably well under a frontal face orientation with open eyes. However, they will fail if the pupils are not bright enough due to oblique face orientations, eye closures, or external illumination interferences. The Kalman filters also fail when a sudden head movement occurs because the assumption of a smooth head motion has not been fulfilled. To overcome this limitation, we propose a modification that uses an adaptive search window whose size is determined automatically based on the pupil position, pupil velocity, and location error. This way, if the Kalman-filtering tracking fails in a frame, the search window progressively increases its size. With this modification, the robustness of the eye tracker is significantly improved, for the eyes can be successfully found under eye closure or an oblique face orientation.

The state vector of the filter is represented as $\boldsymbol{x}_{t}=$ $\left(\boldsymbol{c}_{t}, \boldsymbol{r}_{t}, \boldsymbol{u}_{t}, \boldsymbol{v}_{t}\right)$, where $\left(\boldsymbol{c}_{t}, \boldsymbol{r}_{t}\right)$ indicates the pupil pixel position (its centroid) at time $\boldsymbol{t}$ and $\left(\boldsymbol{u}_{t}, \boldsymbol{v}_{t}\right)$ be its velocity at time $t$ in $c$ and $r$ directions, respectively. Fig. 5 shows an example of the pupil tracker working in a test sequence. The rectangles on the images indicate the search window of the filter while the crosses indicate the locations of the detected pupils. Fig. 5(f) and (g) draw the estimation of the pupil positions for the sequence under test. The tracker is found to be rather robust for different users without glasses, lighting conditions, face orientations, and distances between the camera and the driver. It automatically finds and tracks the pupils even with closed eyes and partially occluded eyes, and can recover from tracking failures. The system runs at a frame rate of 25 frames/s.

The performance of the tracker gets worse when users wear eyeglasses because different bright blobs appear in the image due to the IR reflections in the glasses, as can be seen in Fig. 6 . Although the degree of reflection on the glasses depends on its material and the relative position between the user's head and the illuminator, in the tests, the reflection of the inner ring of LEDs appears as a filled circle on the glasses, which is of the same size and intensity as the pupil. The reflection of the outer ring appears as a circumference with bright points around it and with a similar intensity to the pupil. At the moment, 


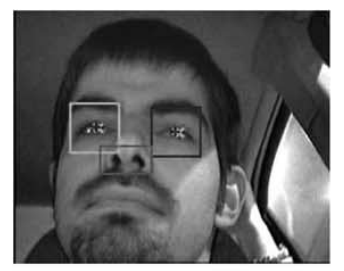

(a)

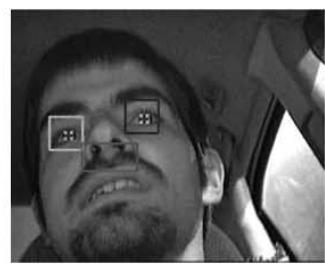

(b)

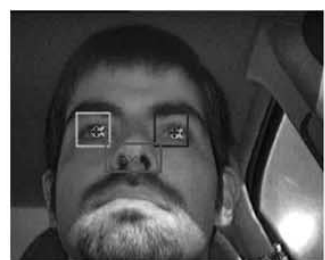

(c)

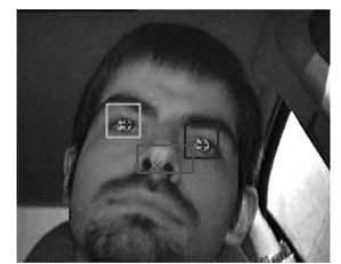

(d)

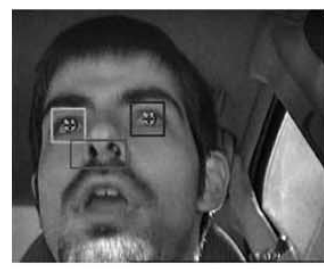

(e)

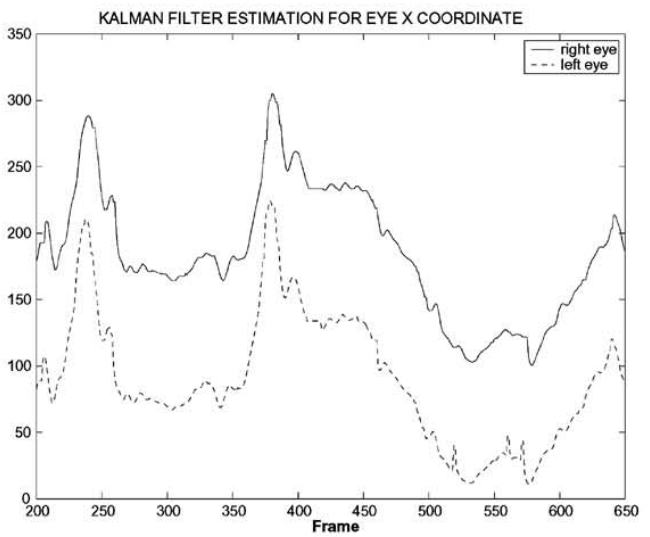

(f)

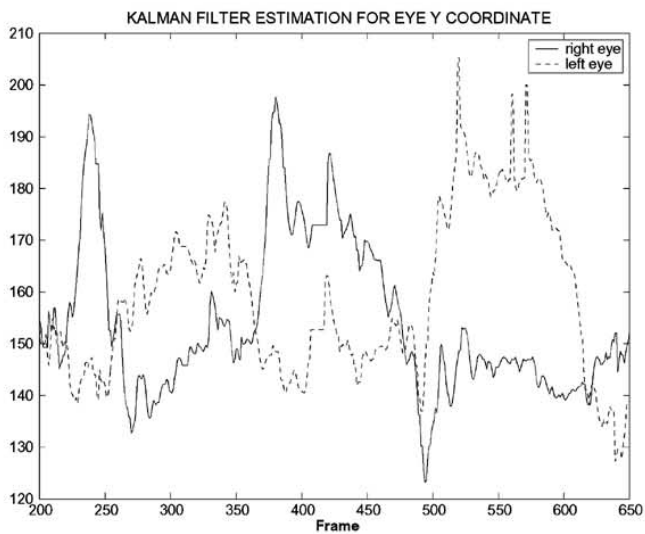

(g)

Fig. 5. Tracking the results for a sequence. (a) Frame 217. (b) Frame 298. (c) Frame 376. (d) Frame 416. (e) Frame 498. (f) Eye x-coordinate estimation. (g) Eye y-coordinate estimation.
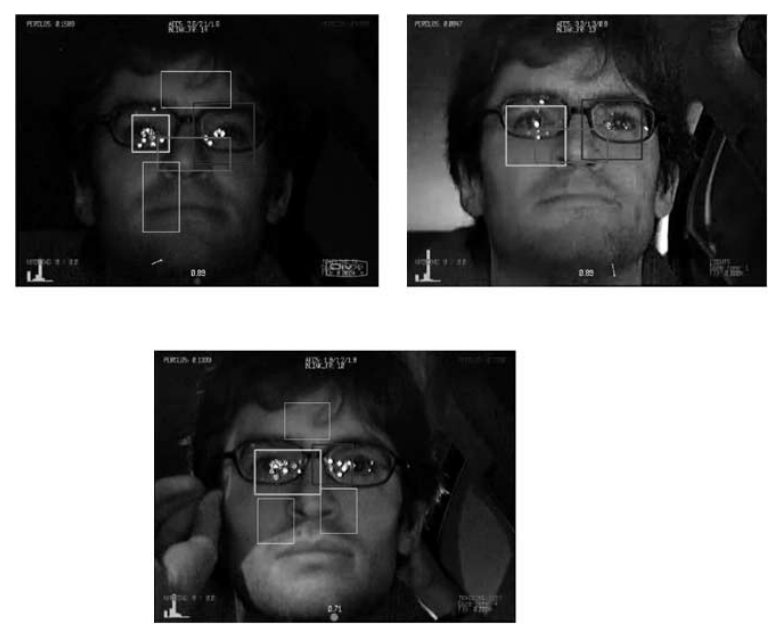

Fig. 6. System working with a user wearing glasses.

we have not applied specific algorithms in order to improve the tracking with glasses; but in the near future, we intend to detect the patterns generated by the outer and the inner rings and remove them from the images. The system was also tested with people wearing contact lenses. In this case, no differences in the tracking were obtained with respect to drivers not wearing them.

\section{Visual Behaviors}

Eyelid movements and face pose are some of the visual behaviors that reflect a person's level of inattention. There are several ocular measures to characterize eyelid movements such as eye closure duration, blink frequency, fixed gaze, eye closure/opening speed, and the recently developed parameter PERCLOS [22], [23]. The last measure indicates the accumulative eye closure duration over time, excluding the time spent on normal eye blinks. It has been found to be the most valid ocular parameter for characterizing driver fatigue [5]. The face pose determination is related to the computation of the face orientation and position, and the detection of head movements. Frequent head tilts indicate the onset of fatigue. Moreover, the nominal face orientation while driving is frontal. If the driver faces in other directions for an extended period of time, it is due to distraction. In this paper, we have measured all the abovementioned parameters in order to evaluate its performance for the prediction of the driver's inattention state, focusing on the fatigue category.

To obtain the ocular measures, we continuously track the subject's pupils and fit two ellipses to each of them using a modification of the algebraic distance algorithm for conics approximation [24], as implemented in the OpenCV library [25]. The degree of eye opening is characterized by the pupil's shape. As the eyes close, the pupils start getting occluded by the eyelids and their shapes get more elliptical. Therefore, we can use the ratio of the pupil ellipse axes to characterize the degree of eye opening. To obtain a more robust estimation of the ocular measures and, e.g., to distinguish between a blink and an error in the tracking of the pupils, we use a finite state machine (FSM), as depicted in Fig. 7. Apart from the init_state, five states have been defined: tracking_ok, closing, closed, opening, and tracking_lost. Transitions between states is achieved from frame to frame as a function of the width-height ratio of the pupils.

The system starts at the init_state. When the pupils are detected, the FSM passes to the tracking_ok state, indicating 


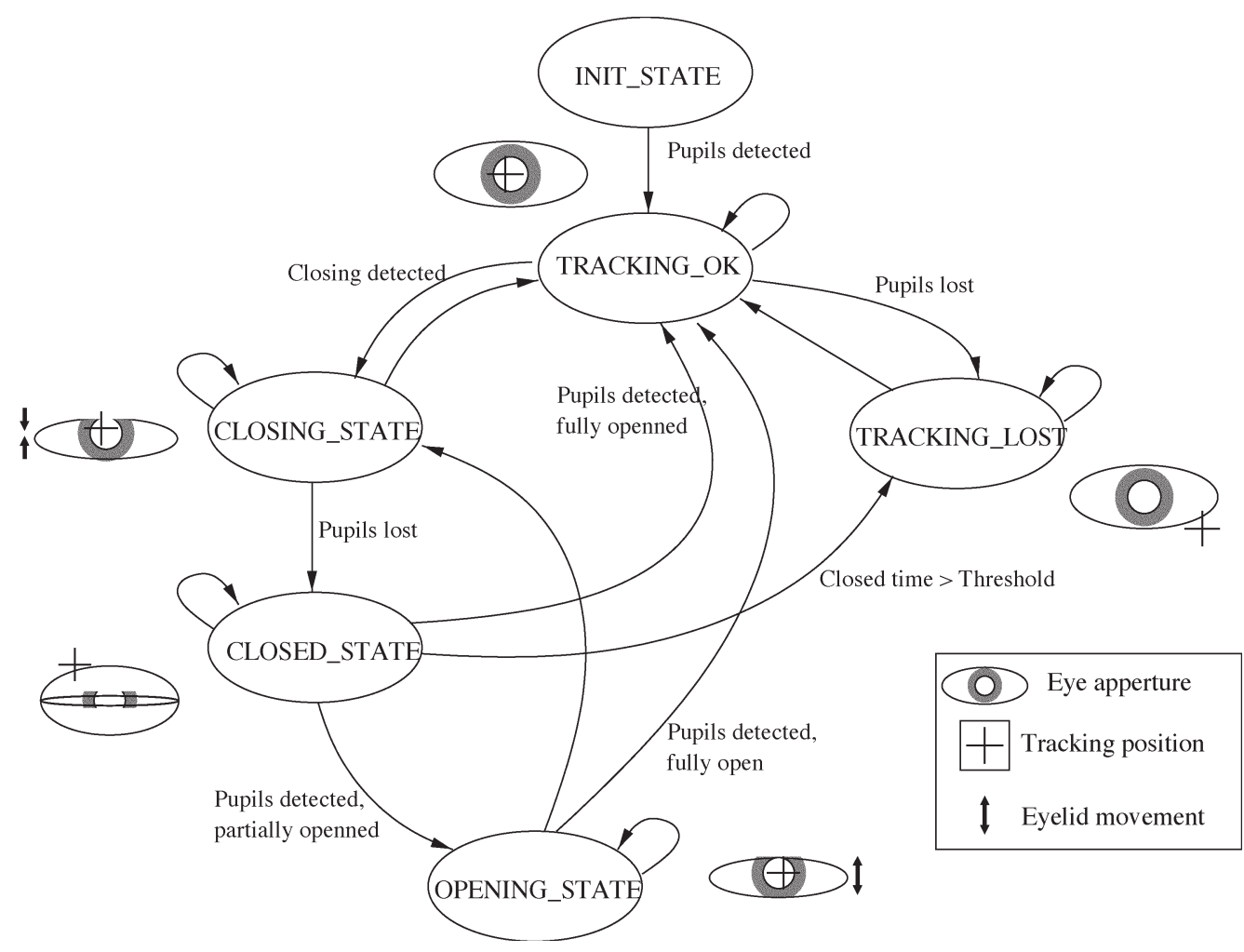

Fig. 7. FSM for ocular measures.

that the pupil's tracking is working correctly. Being in this state, if the pupils are not detected in a frame, a transition to the tracking_lost state is produced. The FSM stays in this state until the pupils are correctly detected again. In this moment, the FSM passes to the tracking_ok state. If the width-height ratio of the pupil increases above a threshold (20\% of the nominal ratio), a closing eye action is detected and the FSM changes to the closing_state. Because the width-height ratio may increase due to other reasons such as segmentation noise, it is possible to return to the tracking_ok state if the ratio does not constantly increase.

When the pupil ratio is above $80 \%$ of its nominal size or the pupils are lost, being in the closing_state, an FSM transition to the closed_state is provoked, which means that the eyes are closed. A new detection of the pupils from the closed_state produces a change to the opening_state or the tracking_ok state, depending on the degree of the opening of the eyelid. If the pupil ratio is between $20-80 \%$, a transition to the opening_state is produced; if it is below $20 \%$, the system passes to the tracking_ok state. Being in the closed_state, a transition to the tracking_lost state is produced if the closed time goes over a threshold. A transition from opening to closing is possible if the width-height ratio increases again. Being in the opening_state, if the pupil ratio is below $20 \%$ of the nominal ratio, a transition to the tracking_ok state is produced.

The ocular parameters that characterize eyelid movements have been calculated as a function of the FSM. The PERCLOS is calculated from all the states, except from the tracking_lost state, analyzing the pupil width-height ratio. We consider that an eye closure occurs when the pupil ratio is above $80 \%$ of its nominal size. Then, the eye closure duration measurement is calculated as the time that the system is in the closed_state. To obtain a more robust measurement of the PERCLOS, we compute this as a running average. We compute this parameter by measuring the percentage of the eye closure in 30-s window. Then, the PERCLOS measurement represents the time percentage that the system is at the closed_state, evaluated in $30 \mathrm{~s}$ excluding the time spent in normal eye blinks. The eye closure/opening speed measurements represent the amount of time needed to fully close the eyes or to fully open the eyes. Therefore, the eye closure/opening speed is calculated as the time period during which the pupil ratio passes from $20 \%$ to $80 \%$ or from $80 \%$ to $20 \%$ of its nominal size, respectively. In other words, the time that the system is in the closing_state or the opening_state, respectively. The blink frequency measurement indicates the number of blinks detected in $30 \mathrm{~s}$. A blink action will be detected as a consecutive transition among the following states: closing, closed, and opening; given that this action was carried out in less than a predefined value. Many physiology studies have been carried out regarding the blinking duration. We have used the recommended value derived in [26], but this could be easily modified to conform to other recommended values. The eye nominal size used for the ocular parameter calculations varies depending on the driver. To calculate the correct value, a histogram of the eye opening degree for the last 2000 frames not exhibiting drowsiness is obtained. The most frequent value of the histogram is considered to be the nominal size. The PERCLOS is computed separately in both eyes and the final value is obtained as the mean of both.

Furthermore, the face pose can be used for detecting fatigue or distraction behaviors among the categories defined for 


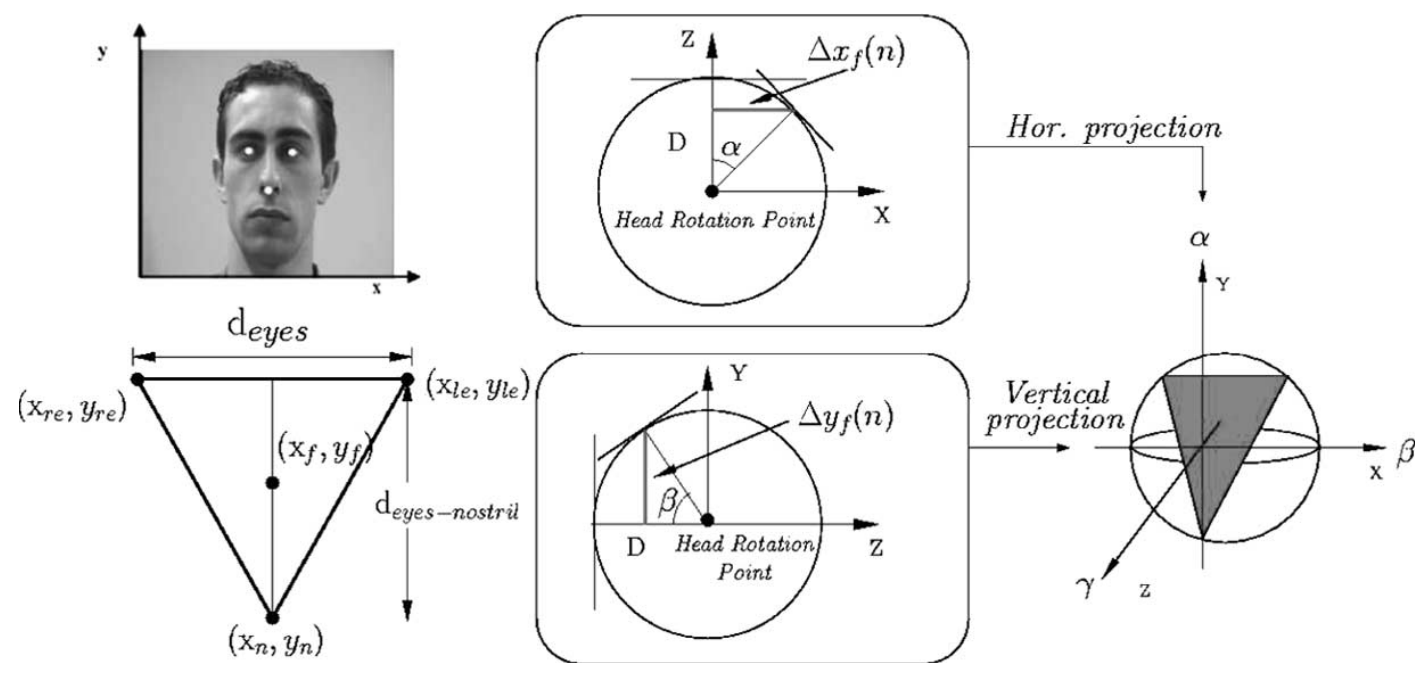

Fig. 8. Recovering the 3-D face pose from 2-D projections.

inattentive states. The normal face orientation while driving is frontal. If the driver's face orientation is in other directions for an extended period of time, it is due to distractions, and if it occurs frequently (in case of various head tilts), it is a clear symptom of fatigue. In our application, the precise degree of face orientation for detecting this behavior is not necessary because face pose in both cases is very different to a frontal one. What we are interested in is to detect whether the driver's head deviates excessively from its nominal position and orientation for an extended period of time or too frequently (nodding detection).

This paper provides a novel solution to the coarse 3-D face pose estimation using a single uncalibrated camera based on the method proposed in [14]. We use a model-based approach for recovering the face pose by establishing the relationship between 3-D face model and its 2-D projections. A weak perspective projection is assumed so that face can be approximated as a planar object with facial features, such as the eyes, nose and mouth, located symmetrically on the plane. We have performed a robust 2-D face tracking based on pupil and the nostril detection on the images. From these positions, the 3-D face pose is estimated. Nostril detection has been carried out in a similar way as that used for the pupils' detection. Nostrils appear in the images as dark pixels surrounded by not so dark pixels (skin), which are then easily detectable. The effect of dark nostrils benefits from the position of the camera in the car.

Initially, we automatically detect a fronto-parallel face view based on the detected pupils and nostrils, as can be seen in Fig. 8. Using the distance between the detected eyes $\left(d_{\text {eyes }}\right)$, the distance between the center of the eyes and the nostrils $\left(d_{\text {eyes-nostrils }}\right)$, eyes and nostrils locations, and some simple anthropometric proportions, the scope and the location of the face in the image is estimated. The detected face region is used as the initial 3-D planar face pose. This method assumes that the distance from the head to the camera remains constant and that head size is relatively constant for all people. As depicted in Fig. 8, with the 2-D position of the eyes and nostril centers, and estimating the distance to the head rotation point (D), we can recover the 3-D face pose using basic projections. We only calculate the vertical and horizontal rotations $(\alpha, \beta)$ because these are the most important features for our purpose. As a function of the calculated rotation from the model and using the speed data of the pupil movements from the Kalman filters, we classify face direction in nine areas: frontal, left, right, up, down, upper left, upper right, lower left, and lower right. Given the initial face image and its pose in the first frame, the task of finding the face location and the face pose in subsequent frames can be implemented as a simultaneous 3-D face pose tracking and face detection. This simple technique works fairly well for all the faces that we have tested, especially with left and right rotations. A more detailed explanation about this method was presented by the authors in [27]. As the goal of this behavior is to detect whether the face pose of the driver is not frontal for an extended period of time, this has been computed using only a parameter that gives the percentage of time that the driver has been looking at the front: over a 30-s temporal window.

Nodding is used to quantitatively characterize one's level of fatigue. Several systems that calculate this parameter from a precise estimation of the driver's gaze have been reported in the literature [16], [20]. However, these systems have been tested in laboratories, but not in real moving vehicles. The noise introduced in real environments makes these systems, based on exhaustive gaze calculation, unable to work properly. In this paper, we present a new technique based on the position and speed data from the Kalman filters used to track the pupils and the FSM. This parameter measures the number of head tilts detected in the last $2 \mathrm{~min}$. We have experimentally observed that in many occasions, nodding follows a pattern along the vertical axis (v) similar to Fig. 9(a). When nodding takes place, the driver closes his or her eyes and the head goes down, touching the chest or the shoulders. If the driver wakes up in that moment, rising his head, the values of the vertical speed of the Kalman filters will describe quite a characteristic curve, as shown in Fig. 9(b). The speed of the Kalman filters changes its sign as the head rises. If the FSM is in the closed_state or in the tracking_lost state and the pupils are detected again, the system saves the speeds of the pupils' trackers for 10 frames. After that, the data is analyzed to find if it conforms to that of nodding. If 


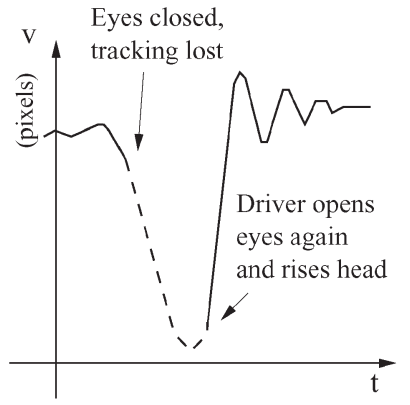

(a)

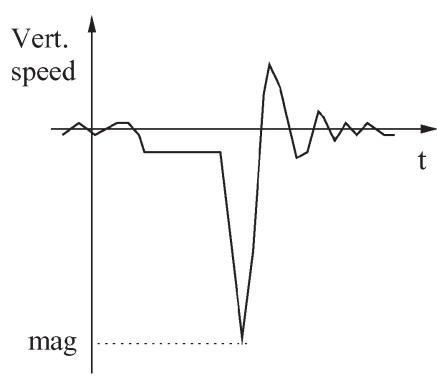

(b)
Fig. 9. Nodding curves.

so, the first stored value is saved and used as an indicator of the "magnitude" of the nodding.

Finally, one of the remarkable behaviors that appears on drowsy drivers is fixed gaze. A fatigued driver loses the focus of the gaze, not paying attention to any of the elements of the traffic. This loss of concentration usually takes place before other sleepy behaviors do, such as nodding. As with the parameter explained above, the existing systems calculate this parameter from a precise estimation of the driver's gaze and, consequently, experience the same problems. In order to develop a method to measure this behavior in a simple and robust way, we present a new technique based on the data from the Kalman filters used to track the pupils.

A driver in a good condition moves his eyes frequently, focusing on the changing traffic conditions, particularly if the road is busy. This has a clear reflection on the difference between the estimated position from the Kalman filters and the measured ones, as can be seen in Fig. 10(a), where fixed gaze behavior is present from 150 to $250 \mathrm{~s}$.

Furthermore, the movements of the pupils of a drowsy driver present different characteristics. Our system monitors the position on the $x$ coordinate. The $y$ coordinate is not used, as the difference between a drowsy and an awake driver is not so clear. The computation of this parameter is based on two temporal windows. In the first one, lasting $2 \mathrm{~s}$, the values in every frame are stored. At the end of it, the mean and the standard deviation values are calculated. If the results for both eyes fall within the predefined limits, that window will be computed as " 1 " and as "0" otherwise, as shown on Fig. 10(b). The second window computes the average of these values ("0" or "1") during the last $60 \mathrm{~s}$ [Fig. 10(c)], this being the parameter passed on to the next stage. This way, the fixed gaze parameter is computed locally in a long period of time, allowing a leeway of the pupil positions over time.

This fixed gaze parameter may suffer from the influence of vehicle vibrations or bumpy roads. Modern cars have reduced vibrations to the point that the effect on the measurements is negligible. The influence of bumpy roads depends on their particular characteristics. If the bumps are occasional, it will only affect few values; these will not represent an important quantity in the overall measurement. On the other hand, if the bumps are frequent and their magnitude is high enough, the system will probably fail to detect this behavior. Fortunately, the probability of a driver falling asleep is significantly lower in very bumpy roads. The results obtained for all the test sequences with this parameter are encouraging. In spite of using the same $a$ priori threshold for different drivers and situations, the detection was always correct. Even more remarkable was the absence of false positives.

\section{Driver Vigilance Computation}

This section describes the method to determine the driver's visual inattention level from the parameters obtained in the previous section. This process is very complicated because several uncertainties may be present. First, fatigue is not observable and it can only be inferred from the available information. In fact, this behavior can be regarded as the result of many contextual variables such as environment, health, and sleep history. To effectively monitor it, a system that integrates evidences from multiple sensors is needed. In the present study, several visual fatigue behaviors are subsequently combined to form an inattentive parameter that can robustly and accurately characterize one's vigilance level. The fusion of the parameters has been obtained using a fuzzy system. We have chosen this technique for its well-known linguistic concept modeling ability. The fuzzy rule expression is close to an expert natural language. A fuzzy system then manages the uncertain knowledge and infers high level behaviors from the observed data. On the other hand, as it is a universal approximator, the fuzzy inference system can be used for knowledge induction processes. The objective of our fuzzy system is to provide a driver's inattentiveness level (DIL) from the fusion of several ocular and face pose measures, along with the use of expert and induced knowledge. This knowledge has been extracted from the visual observation and the data analysis of the parameters in some simulated fatigue behaviors carried out in real conditions (driving a car) with different users. The simulated behaviors have been done according to the physiology study of the U.S. Department of Transportation, presented in [5]. This paper does not delve into the psychology of driver's visual attention; rather, it merely demonstrates that with the proposed system, it is possible to collect the driver information data and infer whether the driver is attentive or not.

The first step in the expert knowledge extraction process is to define the number and nature of the variables involved in the diagnosis process according to the domain expert experience. The next variables are proposed after appropriate study of our system: PERCLOS, eye closure duration, blink frequency, nodding frequency, fixed gaze, and frontal face pose. The eye closing and opening variables are not used in our input fuzzy set because they depend mainly on factors such as segmentation and the correct detection of the eyes and take place in a period of time comparable in length with that of the image acquisition. As a consequence, they are very noisy variables. As our system is adaptive with regards to the user, the ranges of the selected fuzzy inputs are approximately the same for all users. The fuzzy inputs are normalized and different linguistic terms and their corresponding fuzzy sets are distributed in each of them using induced knowledge based on the hierarchical fuzzy partitioning (HFP) method [28]. Its originality lies not in yielding a single partition, but in a hierarchy including partitions with different 


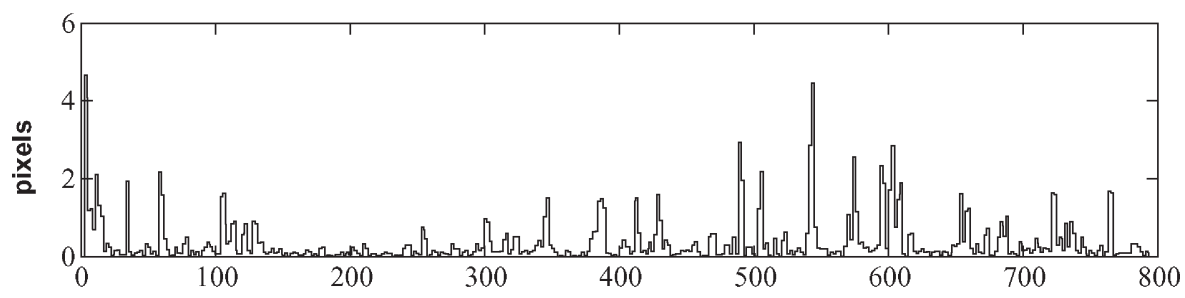

(a)

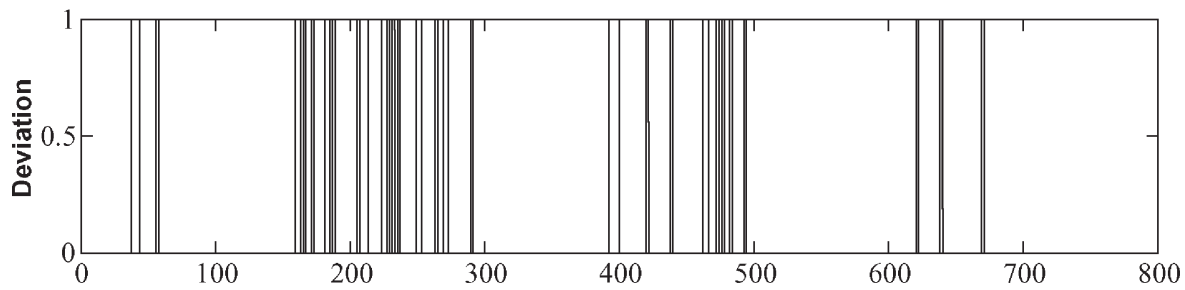

(b)

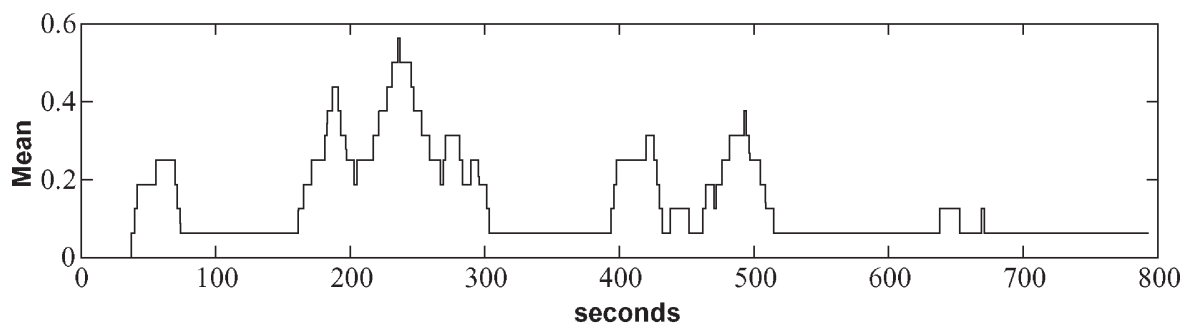

(c)

Fig. 10. Measures for the "fixed gaze" parameter. (a) Difference between real and measured eye position. (b) Label of the 2-s window after deviation on computation. (c) Mean of the deviation value over a 60-s window.

TABLE I

FUZZY VARIABLES

\begin{tabular}{c|c|c|c|c}
\hline Variable & Type & Range & Labels & Linguistic terms \\
\hline \hline PERCLOS & In & {$[0.0,1.0]$} & 5 & small, medium small, medium, medium large, large \\
\hline Eye closure duration & In & {$[1.0-30.0]$} & 3 & small, medium, large \\
\hline Blink freq. & In & {$[1.0-30.0]$} & 3 & small, medium, large \\
\hline Nodding freq. & In & {$[0.0-8.0]$} & 3 & small, medium, large \\
\hline Face position & In & {$[0.0-1.0]$} & 5 & small, medium small, medium, medium large, large \\
\hline Fixed gaze & In & {$[0.0-0.5]$} & 5 & small, medium small, medium, medium large, large \\
\hline DIL & Out & {$[0.0-1.0]$} & 5 & small, medium small, medium, medium large, large \\
\hline
\end{tabular}

resolution levels based on automatic clustering data. Analyzing the fuzzy partitions obtained by HFP, we determined that the best suited fuzzy sets and the corresponding linguistic terms for each input variable are those shown in Table I. For the output variable (DIL), the fuzzy set and the linguistic terms were manually chosen. The inattentiveness level range is between 0 and 1 , with a normal value of up to 0.5 . When its value is between 0.5 and 0.75 , the driver's fatigue is medium, but if the DIL is over 0.75 , the driver is considered to be fatigued, and an alarm is activated. Fuzzy sets of triangular shapes were chosen, except at the domain edges, where they were semitrapezoidal.

Based on the above selected variables, experts state different pieces of knowledge (rules) to describe certain situations connecting some symptoms with a certain diagnosis. These rules are of the form "If condition, Then conclusion," where both the premise and conclusion use the linguistic terms previously defined, as in the following example:

1) IF PERCLOS is large AND Eye Closure Duration is large, THEN DIL is large.
In order to improve the accuracy and system design, the automatic rule generation and its integration in the expert knowledge base were considered. To facilitate such an integration, the generated rules use the readable fuzzy partitions which have already been designed. The process of generating rules from data is called induction. It aims at producing general statements, expressed as fuzzy rules in our case, valid for the whole set from partial observations obtained from some real experiments. As the data are likely to give a good image of interactions, induced rules may yield complementary pieces of knowledge. A lot of methods are available in the fuzzy literature [29]. We restrict our interest to the ones that generate rules that share the same fuzzy sets (Wang and Mendel (WM) [30], fast prototyping algorithm (FPA) [31] and fuzzy decision trees (FDT) [32]). Among them, we chose the FDT with the pruned method (FDT + P) because it produces the best quality, with a more interpretable and accurate knowledge base.

The induced rules with $\mathrm{FDT}+\mathrm{P}$ were integrated into the expert knowledge base. As a result, the rule base consists of 
TABLE II

Length of the Simulated DRowsiness Sequences

\begin{tabular}{c|c|c|c}
\hline Seq. Num. & Drowsiness Behavior time(sec) & Alertness Behavior time(sec) & Total time(sec) \\
\hline \hline 1 & $394(2$ intervals: $180+214)$ & 516 & 910 \\
\hline 2 & $90(1$ interval $)$ & 210 & 300 \\
\hline 3 & 0 & 240 & 240 \\
\hline 4 & $155(1$ interval $)$ & 175 & 330 \\
\hline 5 & $160(1$ interval $)$ & 393 & 553 \\
\hline 6 & $180(1$ interval $)$ & 370 & 550 \\
\hline 7 & $310(2$ intervals: $150+160)$ & 631 & 941 \\
\hline 8 & $842(2$ intervals:390+452) & 765 & 1607 \\
\hline 9 & $210(2$ intervals: $75+135)$ & 255 & 465 \\
\hline 10 & $673(2$ intervals:310+363) & 612 & 1285 \\
\hline
\end{tabular}

94 rules, eight expert rules, and 86 induced ones. During this last step, the fundamental properties of the rule base have to be guaranteed: consistency, lack of redundancy, and interpretability. Both kinds of rules use the same linguistic labels, thanks to the previously defined common universe. Therefore, rule comparison is made at the linguistic level only.

First of all, a consistency analysis [33] of the knowledge base is made in order to detect conflicts at linguistic level. Afterwards, a simplification process is applied with the goal of achieving a more compact knowledge base with a smaller size to improve interpretability, mantaining the accuracy of the original knowledge base. The simplification process is described in detail in [34].

This paper only describes the simplification process results in the real problem under consideration. Please refer to the cited literature for a complete description. The final knowledge base is more compact, with a smaller number of rules that are incomplete and more general, and with a smaller number of labels. We have obtained a rule base with 32 rules, which are easily interpretable. According to these rules, three variables (fixed gaze, PERCLOS and eye closure duration) are crucial for determining driver's fatigue. As an example, two induced rules are shown in the following:

1) IF PERCLOS is small AND Eye Closure Duration is small AND Face Position is medium AND Nodding Frequency is small AND Blink Frequency is medium AND Fixed Gaze is small, THEN DIL is small.

2) IF PERCLOS is medium large AND Eye Closure Duration is medium AND Blink Frequency is medium AND Fixed Gaze is large, THEN DIL is large.

The fuzzy system implementation was done using the licence-free knowledge base configuration tool (KBCT) [35] developed by the intelligent systems group of the Technical University of Madrid (UPM). In the next section, we present the experimental results obtained with this tool with the following basic fuzzy options: minimum operator as the connective AND, maximum as the aggregation method, and center of area as the defuzzification method. All induced rules have the same weight.

\section{EXPERIMENTAL RESULTS}

The goal of this section is to experimentally demonstrate the validity of our system in order to obtain the fatigue behaviors in drivers. First, we show some details about the recorded video sequences used for testing; then, we analyze the parameters measured for one of the sequences. Finally, we present the performance of the detection of each one of the parameters and the overall performance of the system.

\section{A. Test Sequences}

Ten sequences were recorded in real driving situations in a highway and a both-senses road. Each sequence was obtained for a different user. The images were obtained using the system explained in Section III-A. The drivers simulated some drowsy behaviors according to the physiology study of the U.S. Department of Transportation presented in [5]. Each user drove normally, except in one or two intervals where the driver simulated fatigue. The length of the sequences and the fatigue simulation intervals is shown in Table II. All the sequences were recorded at night, except for sequence number 7 that was recorded at daytime, and sequence number 5 that was recorded at sunset. Sequences were obtained with different drivers not wearing glasses, with the exception of sequence 6 , which was recorded for testing the effects of the use of glasses in real driving conditions.

\section{B. Parameter Measurement for One of the Test Sequences}

The system is currently running on a Pentium 4 1.8-Ghz PC with Linux kernel 2.4.24 in real time (25 frames/s) with a resolution of $640 \times 480$ pixels. The average processing time per frame (both even and odd fields) is $11.43 \mathrm{~ms}$. Fig. 11 depicts the parameters measured for sequence number 9 . This is a representative test example with a duration of $465 \mathrm{~s}$ where the user simulates two fatigue behaviors separated by an alertness period. As can be seen, until the 90 -s interval, and between the 195- and 360-s intervals, the DIL parameter is below 0.5, indicating an alertness state. In these intervals, the PERCLOS is low (below 0.15), the eye closure duration is low (below the $200 \mathrm{~ms}$ ), the blink frequency is low (below 2 blinks per 30-s window) and the nodding frequency is zero. These ocular parameters indicate a clear alert behavior. The frontal face position parameter is not 1.0, indicating that the predominant position of the head is frontal, but that there are some deviations near the frontal position, typical of a driver with a high vigilance level. The fixed gaze parameter is low because the eyes of the driver are moving caused by a good alert condition. The DIL parameter increases over the alert 


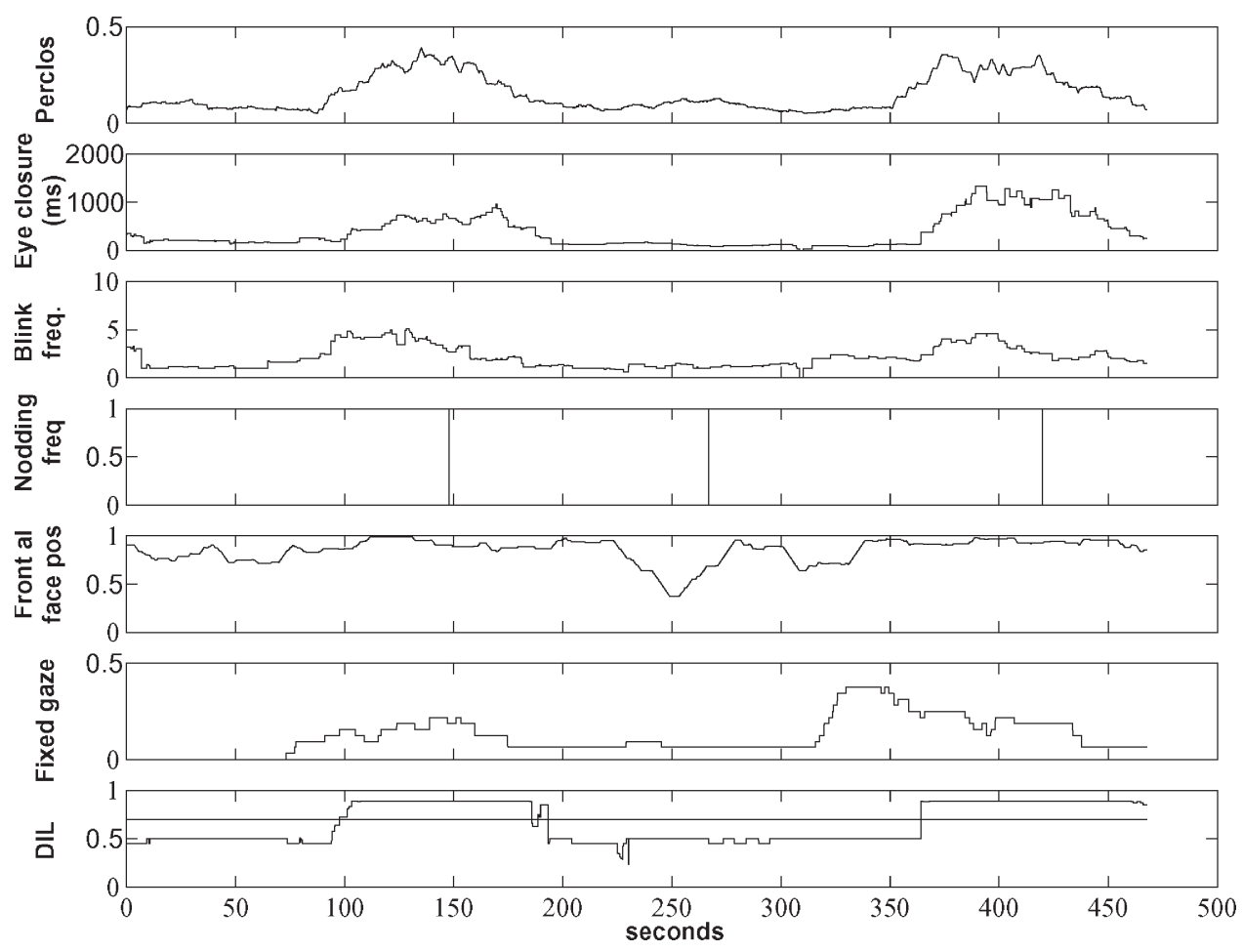

Fig. 11. Parameters measured for test sequence 9.

TABLE III

Parameter Detection Performance

\begin{tabular}{c|c|c|c|c|c|c|c|c|c|c|c}
\hline \multirow{2}{*}{ Parameters } & \multicolumn{7}{|c|}{ Correct percentage per sequence } & \multirow{2}{*}{$\begin{array}{c}\text { Total correct } \\
\text { percentage }\end{array}$} \\
\cline { 2 - 12 } & 1 & 2 & 3 & 4 & 5 & 6 & 7 & 8 & 9 & 10 & \\
\hline \hline PERCLOS & 90 & 90 & 95 & 95 & 95 & 25 & 50 & 95 & 95 & 90 & $93.12 \%$ \\
\hline Eye closure duration & 90 & 80 & 85 & 90 & 85 & 20 & 50 & 80 & 85 & 80 & $84.37 \%$ \\
\hline Blink freq. & 75 & 75 & 85 & 80 & 85 & 0 & 30 & 85 & 80 & 75 & $80 \%$ \\
\hline Nodding freq. & 70 & 60 & 80 & 80 & 60 & 0 & 20 & 70 & 100 & 60 & $72.5 \%$ \\
\hline Face position & 100 & 70 & 85 & 95 & 90 & 0 & 50 & 85 & 90 & 85 & $87.5 \%$ \\
\hline Fixed gaze & 100 & 90 & 95 & 95 & 95 & 20 & 60 & 95 & 95 & 100 & $95.62 \%$ \\
\hline
\end{tabular}

threshold during two intervals (from 90 to $190 \mathrm{~s}$ and from 360 to $565 \mathrm{~s}$ ), indicating two fatigue behaviors. In both intervals, the PERCLOS increases from 0.15 to 0.4 , the eye closure duration goes up to $1000 \mathrm{~ms}$, and the blink frequency parameter increases from two to five blinks. The frontal face position is very close to 1.0 because the head position is fixed and frontal. The fixed gaze parameter value increases to 0.4 due to the narrow gaze in the line of sight of the driver. This last variation indicates a typical loss of concentration and takes place before the other sleepy measurements, as can be observed. Nodding is the last fatigue effect to appear. In the two fatigue intervals, nodding occurs after the increase of the other parameters, indicating a low vigilance level. We must remark that this last parameter is calculated over a temporal window of $2 \mathrm{~min}$, which is the reason why its value remains stable during this time.

This section described an example of the parameter evolution for two fatigue behaviors and one driver. We then analyze the behaviors of other drivers in different circumstances, according to the video tests explained above. The results obtained are very similar to those shown for sequence number 9 . The overall results of the system are explained as follows.

\section{Parameter Performance}

The general performance of the measured parameters for a variety of environments with different drivers and according to the test sequences is presented in Table III. The performance was measured by comparing the system performance to results obtained by manually analyzing the recorded sequences on a frame-by-frame basis. For each parameter, the correct percentage per sequence is depicted. This includes the correct detection and false positives. The last column depicts the total correct percentage for all sequences, excluding sequence number 6 (driver wearing glasses) and sequence number 7 (recorded by day). Therefore, this column shows the parameter detection performance of the system for optimal situations (driver without glasses driving at night). As can be seen, the performance 
gets considerably worse by day and it dramatically deteriorates when drivers wear glasses.

The PERCLOS results are quite good, obtaining a total correct percentage of $93.12 \%$. It has been found to be a robust ocular parameter for characterizing driver fatigue. However, it may fail at times, e.g., when a driver falls asleep without closing her eyes. The eye closure duration performance is a little worse than that of PERCLOS (84.37\%) because the correct estimation of the duration is more critical. The variation of the intensity when the eyes are partially closed with regards to the intensity when they are open complicates the segmentation and detection. This causes the frame count for this parameter to be usually lesser than the real one. These frames are considered as closed time. The measured time is slightly over the real time as a result of the delayed detection. The performance of the blink frequency parameter is about $80 \%$ because some quick blinks are not detected using a frame rate of 25 frames/s. Then, the three parameters are clearly correlated almost linearly, with PERCLOS being the most robust and accurate one.

The nodding frequency results are the worst $(72.5 \%)$ parameter, as the system is not sensitive to noddings in which the driver rises her head and then opens her eyes. To reduce the false positives, the magnitude of the nodding (i.e., the absolute value of the Kalman filter speed), must be over a threshold. In most of the nondetected noddings, the first situation took place, while the second limitation did not have any influence on any of them. The ground truth for this parameter was obtained manually by localizing the noddings on the recorded video sequences. It is not correlated with the three previous parameters and is not robust enough for fatigue detection. Consequently, it can be used as a complementary parameter to confirm the diagnosis established based on other more robust methods.

The evaluation of the face direction provides a measure of alertness related to drowsiness and distractions. This parameter is useful for detecting both the pose of the head out of the front direction and the duration of the displacement. The results can be considered fairly good (87.5\%) for a simple model that requires very little computation and no manual initialization. The ground truth for this case was obtained by manually looking for periods in which the driver was clearly not looking at the front in the video sequences and comparing their length to that of the ones detected by the system. There is no clear correlation between this parameter and the ocular ones for fatigue detection. This is the most important cue in the case of distraction detection. The performance of the fixed gaze monitoring is the best of the measured parameters $(95.62 \%)$. The maximum values reached by this parameter depend on the users' movements and gestures while driving, but a level above 0.05 is always considered to be an indicator of drowsiness. Values greater than 0.15 represent a high inattentiveness probability. This parameter did not have false positives and is largely correlated to the frontal face direction. On the contrary, it is not clearly correlated to the rest of the ocular measurements. The ground truth for this parameter was manually obtained by analyzing eye movements frame by frame for the intervals where a fixed gaze behavior was being simulated. The fixed gaze and PERCLOS have been found to be the best detectable parameters for characterizing driver fatigue.
All the parameters presented above are fused together in the fuzzy system to obtain the DIL for the final evaluation of drowsiness. There is some delay between the moment when the driver starts his fatigue behavior simulation and when the fuzzy system detects it. This is a consequence of the window span used in the parameter evaluation. The correct percentage for this output parameter is very high (98\%). It is higher than the value obtained using only the PERCLOS, for which the correct percentage is about the $90 \%$ for our testbench. This is due to the fact that fatigue behaviors are not the same for different drivers. Therefore, the parameter evolution and absolute values from the visual cues differ from user to user. Another important fact is the delay between the appearance of fatigue and its detection. Each parameter responds to a different stage in the fatigue behavior. For example, the fixed gaze behavior appears before the PERCLOS starts to increase, thus raising the DIL to a value where a noticiable increment of the PERCLOS would raise the alarm in few seconds. This is extensible to the other parameters. Using the PERCLOS alone would require more time to raise an alarm (tens of seconds), especially in drivers for which the PERCLOS increases more slowly. Our system provides an accurate characterization of a standard driver's level of fatigue using multiple visual parameters to resolve the ambiguity present in the information from a single parameter. Additionally, the system performance is very high in spite of the partial errors associated with each input parameter. This was achieved using redundant information. The system performance was evaluated by comparing the intervals where the DIL parameter was above 0.75 to the intervals, manually analyzed over the video sequences, in which the driver simulates fatigue behaviors. This analysis consists of a subjective estimation of drowsiness by human observers based on the Wierwille test [23].

\section{Conclusion and Future Studies}

We developed a nonintrusive prototype computer vision system for the real-time monitoring of a driver's vigilance. It is based on a hardware system for a real-time acquisition of driver's images using an active IR illuminator and the implementation of software algorithms for the real-time monitoring of six parameters that better characterize the fatigue level of a driver. These visual parameters are the PERCLOS, eye closure duration, blink frequency, nodding frequency, face position, and fixed gaze. In an attempt to effectively monitor fatigue, a fuzzy classifier was implemented to merge all these parameters into a single DIL. The monitoring of other inattention categories would be possible using this method. The system is fully autonomous; it can initialize automatically, and reinitialize when necessary. It was tested using different sequences recorded in real driving conditions with different users during several hours. In each sequence, several fatigue behaviors were simulated during the test. The system works robustly at night and for users not wearing glasses, yielding an accuracy percentage close to $100 \%$. The performance of the system decreases during daytime, especially in bright days, and at the moment, the system does not work with drivers wearing glasses. 
The results and conclusions obtained provide an approach to the drowsiness detection problem and will be completed in the future studies with actual drowsiness data. In future studies, we intend to test the system with more users for longer periods of time in order to obtain real fatigue behaviors. With this information, we will be able to generalize our fuzzy knowledge base. Then, we would like to enhance our vision system in order to solve the problems for daytime operation and to improve the system for use with drivers wearing glasses. On the other hand, we plan to add two new sensors (a steering wheel and a lateral position sensor) in addition to the visual information to achieve a correct detection, especially at daytime. Finally, this system could be easily extended to other types of vehicles, such as aircraft, trains, subways, etc., consequently improving safety in transportation systems.

\section{REFERENCES}

[1] Awake Consortium (IST 2000-28062), System for effective assessment of driver vigilance and warning according to traffic risk estimation (AWAKE), Sep. 2001-2004. [Online]. Available: http://www. awake-eu.org

[2] D. Royal, "Volume I-Findings report; national survey on distracted and driving attitudes and behaviours, 2002," The Gallup Organization, Washington, D.C., Tech. Rep. DOT HS 809 566, Mar. 2003.

[3] W. Wierwille, L. Tijerina, S. Kiger, T. Rockwell, E. Lauber, and A. Bittner, "Final report supplement-Task 4: Review of workload and related research," U.S. Dept. Transportation, Washington, D.C., Tech. Rep. DOT HS 808467 (4), Oct. 1996.

[4] J. Healey and R. Picard, "SmartCar: Detecting driver stress," in Proc. 15th Int. Conf. Pattern Recognition, Barcelona, Spain, 2000, vol. 4, pp. 218-221.

[5] A. Kircher, M. Uddman, and J. Sandin, "Vehicle control and drowsiness," Swedish National Road and Transport Research Institute, Linkoping, Sweden, Tech. Rep. VTI-922A, 2002.

[6] Anon, "Perclos and eyetracking: Challenge and opportunity," Applied Science Laboratories, Bedford, MA, 1999. [Online]. Available: http:// www.a-s-l.com

[7] H. Ueno, M. Kaneda, and M. Tsukino, "Development of drowsiness detection system," in Proc. Vehicle Navigation and Information Systems Conf., Yokohama, Japan, 1994, pp. 15-20.

[8] J. Soria, Llega el Piloto Tecnológico, pp. 20-21, Mar./Apr. 2002.

[9] DaimerChryslerAG. (2001, Jun.). The Electronic Drawbar. [Online]. Available: http://www.daimlerchrysler.com

[10] Y. Matsumoto and A. Zelinsky, "An algorithm for real-time stereo vision implementation of head pose and gaze direction measurements," in Proc. IEEE 4th Int. Conf. Face and Gesture Recognition, Grenoble, France, Mar. 2000, pp. 499-505.

[11] T. Victor, O. Blomberg, and A. Zelinsky, "Automating the measurement of driver visual behaviours using passive stereo vision," in Proc. Int. Conf. Series Vision Vehicles (VIV9), Brisbane, Australia, Aug. 2001.

[12] Seeing Machines. (2004, Aug.). Facelab Transport. [Online]. Available: http://www.seeingmachines.com/transport.htm

[13] S. Boverie, J. M. Leqellec, and A. Hirl, "Intelligent systems for video monitoring of vehicle cockpit," in Proc. Int. Congr. and Expo. ITSAdvanced Controls and Vehicle Navigation Systems, Detroit, MI, Feb. 1998, pp. 1-5.

[14] P. Smith, M. Shah, and N. D. V. Lobo, "Determining driver visual attention with one camera," IEEE Trans. Intell. Transp. Syst., vol. 4, no. 4, pp. 205-218, Dec. 2003.

[15] W. Shih and J. Liu, "A calibration-free gaze tracking technique," in Proc. 15th Conf. Patterns Recognition, Barcelona, Spain, 2000, vol. 4, pp. 201-204.

[16] Q. Ji and X. Yang, "Real-time eye, gaze and face pose tracking for monitoring driver vigilance," Real-Time Imaging, vol. 8, no. 5, pp. 357-377, Oct. 2002.

[17] R. Grace, "Drowsy driver monitor and warning system," in Proc. Int. Driving Symp. Human Factors Driver Assessment, Training and Vehicle Design, Aspen, CO, Aug. 2001, pp. 64-69.

[18] A. Polychronopoulos, A. Amditis, and E. Bekiaris, "Information data flow in awake multi-sensor driver monitoring system," in Proc. IEEE Intelligent Vehicles Symp., Parma, Italy, Jun. 2004, pp. 902-906.
[19] L. M. Bergasa, J. Nuevo, M. Sotelo, and M. Vazquez, "Real-time system for monitoring driver vigilance," in Proc. IEEE Intelligent Vehicles Symp., Parma, Italy, Jun. 2004, pp. 78-83.

[20] D. Koons and M. Flicker. (2003). IBM Blue Eyes Project. [Online]. Available: http://almaden.ibm.com/cs/blueeyes

[21] Application Note 1118: Compliance of Infrared Communication Products to IEC 825-1 and CENELEC EN 60825-1, Palo Alto, CA: Agilent Technologies, Inc., 1999.

[22] D. Dinges, "F. Perclos: A valid psychophysiological measure of alertness as assesed by psychomotor vigilance," Federal Highway Administration, Office of Motor Carriers, Indianapolis, IN, Tech. Rep. MCRT-98-006, 1998.

[23] W. Wierwille, S. S. Wreggit, C. L. Kirn, L. A. Ellsworth, and R. J. Fairbanks, "Research on vehicle-based driver status/performance monitoring; development, validation, and refinement of algorithms for detection of driver drowsiness, final report; technical reports \& papers," U.S. Dept. Transportation, Washington, D.C., Tech. Rep. DOT HS 808 247, Dec. 1994.

[24] A. W. Fitzgibbon and R. B. Fisher, "A buyer's guide to conic fitting," in Proc. 6th British Conf. Machine Vision, Birmingham, U.K., 1995, vol. 2, pp. 513-522.

[25] G. Bradski, A. Kaehler, and V. Pisarevsky, "Learning-based computer vision with Intel's open source computer vision library," Intel Technol. J., vol. 9, no. 2, pp. 119-130, May 2005.

[26] L. Nunes and M. Recarte, Cognitive Demands of Hands-Free Phone Conversation While Driving. New York: Pergamon, 2002, ch. F 5, pp. 133-144.

[27] L. Bergasa, R. Barea, E. Lopez, and M. Escudero, "Facial features tracking applied to drivers drowsiness detection," in Proc. 21st IASTED Int. Multi-Conf. Applied Informatics, Innsbruck, Austria, Feb. 2003, pp. 231-235.

[28] S. Guillaume and B. Charnomordic, "A new method for inducing a set of interpretable fuzzy partitions and fuzzy inference systems from data," in Studies in Fuzziness and Soft Computing, vol. 128. Heidelberg, Germany: Springer-Verlag, 2003, pp. 148-175.

[29] S. Guillaume, "Designing fuzzy inference systems from data: An interpretability-oriented review," IEEE Trans. Fuzzy Syst., vol. 9, no. 3, pp. 426-443, Jun. 2001.

[30] L.-X. Wang and J. M. Mendel, "Generating fuzzy rules by learning from examples," IEEE Trans. Syst., Man, Cybern., vol. 22, no. 6, pp. 1414-1427, Nov./Dec. 1992.

[31] P. Glorennec, Algorithmes d'apprentissage pour systémes d'inférence floue. Paris, France: Editions Hermés, 1999.

[32] H. Ichihashi, T. Shirai, K. Nagasaka, and T. Miyoshi, "Neuro-fuzzy id3: A method of inducing fuzzy decision trees with linear programming for maximizing entropy and an algebraic method for incremental learning," Fuzzy Sets Syst., vol. 81, no. 1, pp. 157-167, Jul. 1996.

[33] J. M. Alonso, L. Magdalena, and S. Guillaume, "KBCT: A knowledge extraction and representation tool for fuzzy logic based systems," in Proc. IEEE Int. Conf. Fuzzy Systems, Budapest, Hungary, Jul. 2004, vol. 2, pp. 989-994.

[34] — "A simplification process of linguistic knowledge bases," in Proc. Conf. Eur. Society Fuzzy-Logic and Technology (EUSFLAT), Barcelona, Spain, Sep. 2005, pp. 1045-1050.

[35] J. M. A. Moral, S. Guillaume, and L. Magdalena. (2003). KBCT, Knowledge Base Control Tool. [Online]. Available: http://www.mat.upm.es/ projects/advocate/en/index.htm

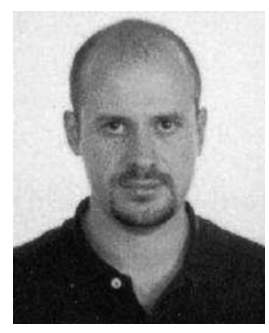

Luis M. Bergasa (M'04-A'05) received the M.S. degree from the Technical University of Madrid, Madrid, Spain, in 1995, and the Ph.D. degree from the University of Alcalá, Madrid, in 1999, all in electrical engineering.

$\mathrm{He}$ is currently an Associate Professor at the Department of Electronics, University of Alcalá. His research interests includes real-time computer vision and its applications, particularly in the field of robotics, assistance systems for elderly people, and intelligent transportation systems. He is the author of more than 60 publications in international journals, book chapters, and conference proceedings.

Dr. Bergasa is a member of the Computer Science Society. 


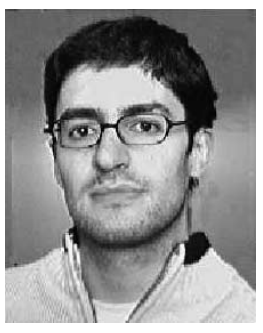

Jesús Nuevo received the M.S. degree in telecommunications engineering from the University of Alcalá, Madrid, Spain, in 2004. He is currently working towards the Ph.D. degree at the same university.

His current research interests include computer vision, autonomous vehicles, pattern recognition, and machine learning.

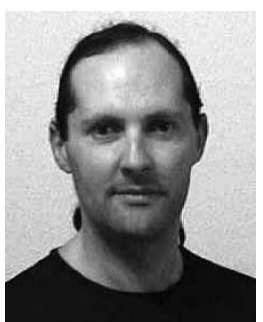

Miguel A. Sotelo (M'02) received the Dr. Ing. degree from the Technical University of Madrid, Madrid, Spain, and the Ph.D. degree from the University of Alcalá, Madrid, all in electrical engineering, in 1996 and 2001, respectively.

From 1993 to 1994, he has been a Researcher at the Department of Electronics, University of Alcala, where he is currently an Associate Professor. His research interests include real-time computer vision and control systems for autonomous and assisted intelligent road vehicles. He is currently serving as an Auditor and an Expert at the Foundation Technological Institute for the Car and Enviroment Security (FITSA) Foundation for R\&D Projects in the domain of automotive applications since September of 2004. He is the author of more than 90 refereed publications in international journals, book chapters, and conference proceedings.

Dr. Sotelo is a member of the IEEE Intelligent Transportation Systems (ITS) Society and is a member of the ITS Spain Committee. He was a recipient of the Best Research Award in the domain of Automotive and Vehicle Applications in Spain in 2002, the 3M Foundation Awards in the category of eSafety in 2003 and 2004, and the Best Young Researcher Award from the University of Alcalá in 2004 .

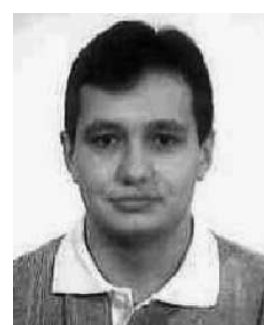

Rafael Barea received the B.S. degree (First Class Honors) from the University of Alcalá, Madrid, Spain, in 1994, the M.S. degree from the Polytechnic University of Madrid, Madrid, in 1997, and the Ph.D. degree from University of Alcalá in 2001, all in telecommunications engineering.

$\mathrm{He}$ is currently an Associate Professor in the Electronics Department, University of Alcalá, where has been Lecturer since 1994. His current research interests include bioengineering, medical instrumentation, personal robotic aids, computer vision, system control, and neural networks. He is the author of many refereed publications in international journals, book chapters, and conference proceedings.

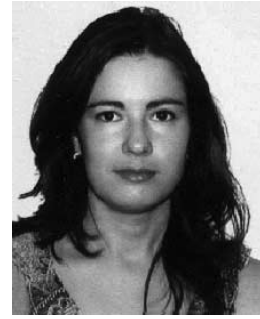

María Elena Lopez received the B.S. degree in telecommunications engineering in 1994, the M.Sc. degree in electronics engineering in 1999, and the $\mathrm{Ph} . \mathrm{D}$. degree in 2004, all from the University of Alcalá, Madrid, Spain.

She has been a Lecturer in the Electronics Department, University of Alcalá since 1995 . Her current research interests include intelligent control and artificial vision for robotics applications. She is the author/coauthor of numerous publications in international journals and conference proceedings in these research lines. 